\title{
Photoionization and electron-ion recombination of Ti I
}

\author{
Sultana N. Nahar \\ Department of Astronomy, The Ohio State University, Columbus, OH 43210 \\ Email: nahar.1@osu.edu, tel: 1-614-292-1888, fax: 1-614-292-2928
}

\begin{abstract}
Study of the inverse processes of photoionization and electron-ion recombination of ( $\mathrm{Ti} \mathrm{I}+\mathrm{h} \nu \leftrightharpoons \mathrm{Ti} \mathrm{II}+\mathrm{e}$ ) using the unified method is reported. The method, based on close coupling (CC) approximation and R-matrix method. subsumes both the radiative recombination $(\mathrm{RR})$ and dielectronic recombination $(\mathrm{DR})$ in a unified manner and provides state-specific and total electron-ion recombination rate coefficients which are self-consistent with the state-specific photoionization cross sections. The present results include state-specific electron-ion recombination rates $\left(\alpha_{R C}(i)\right)$ and partial photoionization cross sections $\left(\sigma_{P I}(i)\right)$ leaving the ion in the ground state of 813 bound states with $\mathrm{n} \leq 10$ and $l \leq 9$ of Ti I. Various features of state-specific and total electron-ion recombination with temperature, and the corresponding photoionization cross sections with energies are discussed with illustrations. Due to closely lying excited states near the ground state of the core, photoionization cross sections show presence of narrow Rydberg resonances in low energy region near the ionization threshold. Many excited states also show broad and enhanced Seaton resonances due to PEC (photo-excitation-of-core) which contribute to the high temperature recombination. The total recombination rate coefficient is found to show a low hump around temperature $280 \mathrm{~K}$ and a high dielectronic recombination peak at temperature $25,000 \mathrm{~K}$. Total spectrum of recombination cross sections and rates with photoelectron energy are also presented for experimental observation. Calculations were carried out using a $\mathrm{CC}$ wave function expansion of 36 states of the core ion Ti II. The large set of data for recombination rates and partial photoionization cross sections with resonances should provide a complete and accurate modelings of plasmas.
\end{abstract}

Keywords: Photoionization and electron ion recombination, state-specific rates of Ti I, unified method, recombination spectrum

32.10-f,95.30.Ky,32.80-t

Email addresses: nahar.1@osu.edu (Sultana N. Nahar), Tel:1-614-292-1888, Fax: 1-292-2928 (Sultana N. Nahar)

URL: http://www. astronomy.ohio-state.edu/ nahar (Sultana N. Nahar)

Preprint submitted to New Astronomy

October 28, 2015

(C) 2015. This manuscript version is made available under the Elsevier user license http://www.elsevier.com/open-access/userlicense/1.0/ 


\section{Introduction}

The earlier report on Ti I focused on the features of total photoionization cross sections of the ground and large number of excited states and benchmarking with experiment [1]. The present study is on partial photoionization cross sections, leaving the residual ion in the ground state, of a large number of bound states with $n \leq 10$ and $l \leq 9$ and the corresponding state-specific recombination rate coefficients. Very little data for photoionization cross sections and recombination rate coefficients are available for modeling of astrophysical and laboratory plasmas. Hahn [2] compiled available RR rates and used Z scaled Coulombic representation and DR rates obtained using empirical fit of a number of Ti ions with core electrons going up to 12 . No work on Ti I has been found.

Ti I lines has been used for synthetic spectra as well as for abundances as they are seen in the spectra of the sun (.e.g. [4], red giant stars (RGS) and in cool M stars (e.g. [5, 6]). The J-band spectra of RSGs are dominated by strong and isolated atomic lines of iron, titanium, silicon [7]. The abundance provides information on flux absorption distribution of the outer layers. However, analysis requires accurate data for radiative processes of photoionization and electron-ion recombination. In determination of $\mathrm{Ti}$ I abundances in the sun and four metal poor late type stars with hydrogenic photoionization cross section, Bergemann [4] finds that the NLTE model for Ti I does not perform well for the metal-poor stars, overestimating NLTE effects in the atmospheres of dwarfs, but underestimating overionization for giants.

The work, carried out under the Iron Project (IP) [8], uses the unified method $[9,10]$ for the total electron-ion recombination that implements ab initio close coupling (CC) approximation and R-matrix method developed under the Opacity Project (OP) [11] and the IP [8].

\section{Theory}

The inverse processes of photoionization and electron-ion recombination can be direct as

$$
h \nu+X^{+} \leftrightharpoons e+X^{++},
$$

where the recombination is radiative recombination (RR). However, depending on the energy they also proceed via an intermediate doubly excited state, as

$$
e+X^{++} \leftrightharpoons\left(X^{+}\right)^{* *} \leftrightharpoons\left\{\begin{array}{c}
a) e+X^{++}(A I) \\
b) h \nu+X^{+}(D R)
\end{array}\right.
$$

where the state known as the autoionizing state breaks either to autoionization (AI) where the electron goes free or to dielectronic recombination (DR) where the electron is captured by emission of a photon (e.g. [12]). The inverse of DR is photoionization. An autoionizing state forms naturally when photon energy matches to that of an autoionizing state of a Rydberg series, $\left(S_{i} L_{i} \pi_{i}\right) \nu l$ where 
$S_{i} L_{i} \pi_{i}$ is an excited state of the core, the outer electron is in excited orbital $\nu l$ where $\nu$ is the effective quantum number belonging to excited core state. Autoionizing state introduces a resonance in the process. Such a resonance can form naturally in calculations by including the core excitations in the wave function, as treated in close coupling (CC) approximation.

In the $\mathrm{CC}$ approximation the atomic system of the core and interacting electrons is represented by $(\mathrm{N}+1)$ electron system where the 'core' of N-electrons is interacting with the $(\mathrm{N}+1)$ th electron. The $(\mathrm{N}+1)$ th electron is bound if the energy $\mathrm{E}$ is negative and in the continuum if it is positive. The total wave function, $\Psi_{E}$, in a symmetry $S L \pi$ is represented by an expansion as

$$
\Psi_{E}(e+i o n)=A \sum_{i} \chi_{i}(\text { ion }) \theta_{i}+\sum_{j} c_{j} \Phi_{j}
$$

where the first term contains core ion eigenfunction, $\chi_{i}$ coupled with the $(\mathrm{N}+1)$ th electron function, $\theta_{i}$ and the sum is over the ground and excited states of the core. The $(\mathrm{N}+1)$ th electron with kinetic energy $k_{i}^{2}$ is in a channel labeled as $S_{i} L_{i} \pi_{i} k_{i}^{2} \ell_{i}(S L \pi)$. In the second sum, the $\Phi_{j}$ s are bound channel functions of the $(\mathrm{N}+1)$-electrons system that account for short range correlation and the orthogonality between the continuum and the bound electron orbitals. Substitution of $\Psi_{E}(e+i o n)$ expansion in the Schrodinger equation

$$
H_{N+1} \Psi_{E}=E \Psi_{E}
$$

leads to a set of coupled equations that are solved using the R-matrix method. The solution is a continuum wave function, $\Psi_{F}$, for an electron with positive energies $(\mathrm{E}>0)$, or a bound state, $\Psi_{B}$, at a negative total energy $(\mathrm{E} \leq 0)$. The details of the R-matrix method in the CC approximation can be found in, e.g. $[13,14,15,12]$. Present calculations are carried out in LS coupling approximation, as in the earlier work [1] where the Hamiltonian of the $(\mathrm{N}+1)$ electrons system is given by

$$
H_{N+1}=\sum_{i=1}^{N+1}\left\{-\nabla_{i}^{2}-\frac{2 Z}{r_{i}}+\sum_{j>i}^{N+1} \frac{2}{r_{i j}}\right\},
$$

Transition matrix elements for photoionization and electron-ion recombination, $T_{B F}=<\Psi_{B}\|\mathbf{D}\| \Psi_{F}>$ where $\mathbf{D}=\sum_{i} r_{i}$ is the dipole operator and the sum is over the number of electrons, can be calculated using the bound and continuum wave functions. Taking the modulus squared, $T_{B F}$ is reduced to generalized line strength as

$$
\mathbf{S}=\left|\left\langle\Psi_{j}\|\mathbf{D}\| \Psi_{i}\right\rangle\right|^{2}=\left|\left\langle\psi_{f}\left|\sum_{j=1}^{N+1} r_{j}\right| \psi_{i}\right\rangle\right|^{2},
$$

where $\Psi_{i}$ and $\Psi_{f}$ are the initial and final state wave functions. The photoionization cross section $\left(\sigma_{P I}\right)$ is proportional to the generalized line strength (e.g. 
$[12])$,

$$
\sigma_{P I}=\frac{4 \pi^{2}}{3 c} \frac{1}{g_{i}} \omega \mathbf{S},
$$

where $g_{i}$ is the statistical weight factor of the bound state and $\omega$ is the incident photon energy. The complex resonant structures in photoionization cross sections result from couplings between the continuum channels and bound channels in transition matrix at electron energies $k_{i}^{2}$ corresponding to autoionizing states of the Rydberg series, $S_{i} L_{i} \pi_{i} \nu \ell$.

Recombination cross sections, $\sigma_{R C}$, can be obtained from detailed partial photoionization cross section using the Milne relation (e.g. [12]),

$$
\sigma_{R C}=\sigma_{P I} \frac{g_{i}}{g_{j}} \frac{h^{2} \omega^{2}}{4 \pi^{2} m^{2} c^{2} v^{2}} .
$$

where $g_{j}$ is the statistical weight factor of the recombined state and $v$ is the photoelectron velocity. The recombining ion is assumed to be in the ground state. The state-specific recombination rate coefficient of state $i$ is obtained by averaging the recombination cross sections as

$$
\alpha_{R}(i ; T)=\int_{0}^{\infty} v f(v) \sigma_{R C}(i) d v,
$$

where $f(v)$ is the Maxwellian electron distribution function,

$$
f(v)=\frac{4}{\sqrt{\pi}}\left(\frac{m}{2 k T}\right)^{3 / 2} v^{2} e^{-\frac{m v^{2}}{2 k T}}
$$

The detailed autoionizing structures in $\sigma_{P I}$ are integrated through $\sigma_{R C}$ and hence corresponds to inclusion of both the RR and DR in an unified and $a b$ initio manner. The total recombination rate can be obtained from sum of these individual rates, that is, $\alpha_{R}(T)=\sum_{i} \alpha_{R}(i, T)$ where the sum is over infinite number of recombined states.

The unified method divides the infinite recombined states into two groups: group (A) states with $n \leq n_{o}$, and group (B) states with $n_{o}<n \leq \infty$, with $n_{o} \sim 10$. The recombination rates coefficients of group $(\mathrm{A})$ states are obtained from detailed photoionization cross sections as described above. Recombination into group (B) states, which are in a small energy range, is dominated by DR via high-n resonances belonging to various excited core thresholds while the background recombination, that is, RR is negligibly small. The precise DR theory by Bell and Seaton [16] was extended by Nahar and Pradhan $[9,10]$ for DR collision strengths, $\Omega(D R)$, as

$$
\Omega(D R)=\sum_{S L \pi} \sum_{n} \frac{1}{2}(2 S+1)(2 L+1) P_{n}^{S L \pi}(D R) .
$$

where $P_{n}^{S L \pi}(\mathrm{DR})=\left(\mathbf{1}-\mathcal{S}_{e e}^{\dagger} \mathcal{S}_{e e}\right)_{n}$ is the DR probability in entrance channel $n$. $\mathcal{S}_{\text {ee }}$ is the electron scattering matrix including radiation damping. Calculations for $\Omega(D R)$ are carried out in the CC approximation using the same wave 
function expansion used for photoionization cross sections and hence are selfconsistent with $\sigma_{P I}$. The recombination cross section is then obtained as (e.g. $[12])$,

$$
\sigma_{R C}(D R)=\frac{\pi}{g_{i} k^{2}} \Omega(D R) a_{o}^{2}
$$

$\Omega(D R)$ is summed over all contributing symmetries $S L \pi$. The total DR contributions of $\mathrm{n}>10$ states is usually much smaller than that for $\mathrm{n}<10$ states except at the DR peak where it can still be smaller but of the same order. The RR 'background' contributions from the high-n group (B) states $n_{o}<n \leq \infty$, obtained in hydrogenic approximation [17], to the total recombination rate are typically negligible except at very low temperatures where electron energies are not high enough for core excitations.

Total electron-ion recombination spectrum with photoelectron energy is of considerable interest as it reveals resonant features and is observable experimentally. The unified total recombination rate coefficients with photoelectron energy, $\alpha_{R}(E)$, can be obtained as

$$
\alpha_{R}(E)=v \sigma_{R C}(E)
$$

where $v$ is the photoelectron energy and $\sigma_{R C}(E)$ summed cross sections of all symmetries.

\section{Computation}

The partial photoionization cross sections of Ti I were computed using the same wave function expansion of 36 states of core ion Ti II as in [1]. Details of the R-matrix calculation are also similar to those as described in [1] except partial cross sections leaving the residual ion in the ground state are computed.

State-specific recombination rate coefficients for all bound states with $n \leq 10$ of group (A) are computed using the code RECOMB [17] and are added together for the total. The contributions of high-n states below the target thresholds of group (B) are obtained from total DR collision strength $\Omega(D R)$ using code STGFDR [10]. The A-values or radiative decay rates for transitions from dipole allowed states to the ground state $3 d^{2} 4 s\left({ }^{4} F\right)$ of the core needed for $\Omega(D R)$ (see [10]) were obtained using the atomic structure code SUPERSTUCTURE [18]. The set of 36 core states [1] of Ti I wave function has six possible dipole allowed transitions. These transitions, along with their A-values and energy positions relative to the ground state, are given in Table 1.

The background (non-resonant) RR contribution from the high-n states $(10<n \leq \infty)$ is included as the "top-up" part [17]. The total unified recombination rate coefficients, $\alpha_{R C}(E)$, cross sections $\sigma_{R C}$ with photoelectron energy, and rate coefficients $\alpha_{R C}(T)$ with temperature were obtained by adding contributions from all $\mathrm{n} \leq \infty$ using program RECXS (.e.g. [3]). 


\section{Results and Discussions}

The partial photoionization cross sections leaving the residual ion in the ground state and electron-ion recombination cross sections and rate coefficients of Ti I are discussed in separate sections below. The choice of a large wave function expansion for Ti I was based on the dipole allowed excitations at high energies of the core ground state as these introduce broad resonances and enhanced background that can have significant contributions to electron-ion recombination.

Ti I has large number of bound states, a total of 813 with $\mathrm{n} \leq 10,0 \leq l \leq$ 9 that couple to the core ground state, $3 d^{2} 4 s\left({ }^{4} F\right)$, and hence contribute to the electron-ion recombination. This number is smaller than 908 bound states with same $\mathrm{n}$ and $l$ as reported in [1]. These additional states are singlets which do not couple to core ground state and hence are not relevant here. Photoionization cross sections and state-specific recombination rate coefficients of all 813 state are presented.

\subsection{Partial photoionization cross sections}

Electron-ion recombination rate coefficients require partial photoionization cross sections $\sigma_{P I}(g, n L S)$ where the core ion is left in the ground state. Important features in $\sigma_{P I}(n L S)$ that affect the recombination cross sections are illustrated below.

States of the atom play different roles in photoionization and electron-ion recombination rates because of the difference in energy positions of resonances and structure of the background cross section with energies. Figure 1 presents partial $\sigma_{P I}$ of (a) ground $3 d^{2} 4 s^{2}\left({ }^{3} F\right)$ and (b) excited $3 d^{2} 4 s^{2} F 4 p\left(y^{3} F^{o}\right)$ states of Ti I illustrating their dominance. The low energy region of each state is filled with strong and dense resonances belonging to various closely lying core states near the ground state and hence contribute considerably to recombination rates. In contrast to the ground states, the excited $3 d^{2} 4 s^{2} F 4 p\left(y^{3} F^{o}\right)$ state will continue to contribute considerably in the high temperature recombination because of the slow decay of the background photoionization cross sections.

Excited states with single outer electron usually have the characteristic broad and enhanced Seaton or PEC (photo-excitation-of-core) resonances [19, 12], that can make important contributions, particularly at high temperature recombination. Figure 2 presents partial photoionization cross sections of two such excited states, (a) $3 d^{4} 4 s^{2} F 4 p\left(z^{3} G^{o}\right)$, (b) $3 d^{2} 4 s^{4} F 5 s\left(b^{5} F\right)$. A Seaton resonance is formed when the core ion absorbs the photon matching the transition energy for the ground state excitation to a dipole allowed one during which the outer electron remains as a spectator. The excitation leads to ionization as the core drops down to the ground state. The phenomenon is inverse to DR, and manifests in a resonance, often wider, at the excited core threshold. Table of 36 core states [1] indicates, as presented in Table 1 here, six possible dipole allowed transitions and hence six possible PEC resonances around energies of $0.28,0.37$ and 0.48 Ry. The Seaton or PEC resonances at these positions are pointed by arrows in Figure 2. These resonances form at the core excitation thresholds 
regardless of the ionization energy of the state. The figure shows that the first three PEC positions are below the ionization energy of state $3 d^{4} 4 s^{2} F 4 p\left(z^{3} G^{o}\right)$ (panel a) and hence Seaton resonances of only the higher ones are seen in its $\sigma_{P I}$. However the first three core excitations have introduced a huge Seaton resonance with background enhanced by orders of magnitude in $\sigma P I$ of $b^{5} F$ (panel b). The next three Seaton resonances are weaker relative to the first three.

\subsection{Total and state-specific recombination rate coefficients}

State specific recombination rate coefficients of all 813 bound states of Ti I presented here can be treated as the total rate for each individual state since for each one, the contributions of both the RR and DR are included by the unified method.

Figure 3 shows illustrative examples of characteristic features of state-specific recombination rate coefficients of the ground state (a) $3 d^{2} 4 s^{2}\left(a^{3} F\right)$, and a number of excited states, (b) $3 d^{3}{ }^{4} F 4 p\left(y^{3} D^{o}\right)$, (c) $3 d^{2} 4 s^{2} F 4 p\left(y^{3} F^{o}\right)$, (d) $3 d^{2} 4 s^{4} F 4 p\left(z^{3} F^{o}\right)$, (e) $3 d^{2} 4 s^{4} F 4 p\left(z^{3} G^{o}\right)$. (f) $3 d^{2} 4 s^{2} F 4 p\left(y^{3} G^{o}\right)$, (g) $3 d^{3} 4 s\left(a^{3} H\right)$ of Ti I. These states dominate electron-ion recombination at different temperatures. Some states show multiple bumps below the temperature of a million degree. The differences in them arise from positions of strong resonances and background structure of photoionization cross sections of the states as explained above. While resonances cause the DR bumps, the background cross sections affects the way recombination rate decays with temperature.

It is often assumed that the ground state of an ion contribute the most to electron-ion recombination. However, the contribution of an excited state may exceed to that of the ground state depending on temperature region. Table 2 lists dominant states, in order of their contributions to the total rate, at four different temperatures. As the table shows, different sets of states dominant the recombination at temperatures of $300,2500,10^{4}$, and $10^{5} \mathrm{~K}$.

While all state-specific rate coefficients are available on-line, Table 3 presents $\alpha_{R C}(n L S, T)$ in the temperature range of $10-10^{9} \mathrm{~K}$ of a number of most dominant contributing states as needed for population modeling. The present state specific rates should be accurate for most of the temperature range except at lower temperature where uncertainty may be introduced from resolution and positions of the resonances. The resolution becomes less significant at higher temperature since they are damped out by the exponential factor in the $\alpha_{R}(n L S)$ integral. Based on the accuracy of the R-matrix method, energy comparison, and inclusion of interference effects, these rates are expected to be accurate within $15-30 \%$.

Features of the total recombination rate coefficients with temperature, $\alpha_{R}(T)$, of $\mathrm{Ti}$ I are presented in Figure 4 and numerical values are presented in Table 4. As expected, starting with a high rate at very low temperature due to dominance of RR, $\alpha_{R}(T)$ decreases with increasing temperature. However, it slows down some forming a small hump around $280 \mathrm{~K}$ due to low energy near threshold resonances in the photoionization cross sections. It continues to decrease until about $6300 \mathrm{~K}$ before rising again to form a broad structure due to dominance 
by DR which reaches the peak at $25,000 \mathrm{~K}$ beyond which it decays down fast. Being an neutral atom, fine structure effects is not expected to be significant for Ti I. One advantage of the unified method over the separate computation of RR and DR rates and adding them for the total is consideration of interference between RR and DR. The effect becomes more important when $\alpha_{R C}$ goes through the minimum, such as arid $6300 \mathrm{~K}$ for Ti I, where unified method provides the natural curve of interference instead of sharp distinction of $R R$ and $D R$ rates obtained separately.

Total recombination rate coefficient with photoelectron energy, $\alpha_{R}(E)$, of (e $+\mathrm{Ti}$ II $\rightarrow$ Ti I) can reveal detailed resonant structures in recombination process and the resonant part can be observed in storage rings. Figure 5 presents total unified $\alpha_{R}(E)$ of Ti I with energy. Panel (a) presents the summed contributions of all triplets and quintets states of Ti I to the total recombination cross section, and panel (b) presents the total recombination rates with photoelectron energy. The resonant DR structures at the very low energy are formed by the low energy resonances in photoionization. The arrows in Figure 5 points the core thresholds such as, $3 d^{2} 4 p\left({ }^{4} G F D^{o}\right)$, where resonances converged forming DR peaks. Immediately beyond a DR peak, recombination drops almost to zero as the trapped photons are released.

\section{Conclusion}

Electron-ion recombination and photoionization of $(\mathrm{h} \nu+\mathrm{Ti} \mathrm{I} \leftrightarrow \mathrm{e}+\mathrm{Ti}$ II $)$ are studied in detail. Unified method has provided self-consistent sets of photoionization cross sections and recombination rates. Photoionization shows extensive low energy Rydberg resonances and wide Seaton resonances, and variations in the background cross sections. Unified method subsumes both RR and DR and their interference for the total state-specific and cumulative total recombination rates by including these resonances. Due to resonance positions and background features in photoionization, the state-specific recombination rate coefficients shows noticeable one or multiple DR bumps. We find that the dominant contributor states depend on temperature and ground state is not the most dominant state. The total recombination rate coefficient shows a hump around $280 \mathrm{~K}$ and a high DR peak at around 25,000 K. Total recombination cross section and rate coefficient with photoelectron energy are also presented for future experiment. The state-specific and total recombination rate coefficients $\alpha_{R}(T)$, are reported for the first time. State specific recombination rate coefficients and partial photoionization cross sections are presented for 813 bound states with $\mathrm{n} \leq 10$ and $l \leq 9$ of Ti I. The present cross sections and recombination rates are expected to be of high accuracy of about 15-30\% based on (i) inclusion of large number of core excitations, (ii) higher resolution for resonances, (ii) consideration of large number configurations, and good agreement of energies with those listed at NIST and photoionization cross sections with experiment [1]. These should provide a more complete modeling of astrophysical and laboratory applications, particularly for low temperature plasmas, 
where $\mathrm{Ti}$ I exist in various cool stars and in determination of abundance of $\mathrm{Ti}$ in these objects.

The complete sets of photoionization cross sections and recombination rate coefficients of Ti I are available on-line at NORAD-Atomic-Data (NaharOSURadiativeAtomicData) website at:

http:/www.astronomy.ohio-state.edu/ nahar/nahar_radiativeatomicdata

Acknowledgments

This work was supported partially by NSF AST-1312441 and AST-1109088. The computational work was carried out at the Ohio Supercomputer Center (OSC) in Columbus Ohio.

\section{References}

[1] S.N. Nahar, New Ast. 38, 16-22 (2015)

[2] Y. Hahn, Phys Scr. Vol. T37, 53-61 (1991)

[3] S.N. Nahar, J. Quant. Spec. Rad. Transfer 109, 2731-2742 (2008)

[4] M. Bergemann, Mon. Not. R. Astron. Soc. 413, 21842198 (2011)

[5] P.C. Keenan, Astron Soc. Pacific 94, 299-303 (1982)

[6] J.A. Valenti, N. Piskunov, C.M. Johns-Krull, ApJ 498, 851 (1998)

[7] M. Bergemann, R-P Kudritzki, B. Plez, B. Davies, K. Lind, Z. Gazak, ApJ $751,156(2012)$

[8] D.G. Hummer, K.A. Berrington, W. Eissner, A.K. Pradhan, H. Saraph, J.A. Tully, Astron. Astrophys. 279:298-309 (1993)

[9] S.N. Nahar, A.K. Pradhan, Phys. Rev. Lett 68, 1488 (1992)

[10] S.N. Nahar, A.K. Pradhan, Phys. Rev. A 49, 1816 (1994)

[11] The Opacity Project Team .The Opacity Project, Vol 1, 1995, Vol. 2, 1996, Institute of Physics Publishing

[12] A.K. Pradhan and S.N. Nahar, Atomic Astrophysics and Spectroscopy (Cambridge University press, 2011)

[13] P.G. Burke, W.D. Robb, Adv. At. Mol. Phys. 11, 143-214 (1975)

[14] M.J. Seaton, J. Phys. B 20, 6363-6378 (1987)

[15] K.A. Berrington, P.G. Burke, K. Butler, M.J. Seaton, P.J. Storey, K.T. Taylor, Y. Yu, J. Phys. B 20,6379-6397 (1987)

[16] R.H. Bell, M.J. Seaton, J. Phys. B 18, 1589 (1985) 
Table 1: Radiative decay rates, $A_{j i}$, for dipole allowed transitions from various excited $f$ states to the ground state $3 d^{2} 4 s\left({ }^{4} F\right)$ of core Ti II. E is the energy position of the excited state relative to the ground state.

\begin{tabular}{ccclcc}
\hline $\begin{array}{c}\text { Core } \\
\text { State:f }\end{array}$ & $\begin{array}{c}A_{f g} \\
\left(s^{-1}\right)\end{array}$ & $E(R y)$ & $\begin{array}{c}\text { Target } \\
\text { State:f }\end{array}$ & $\begin{array}{c}A_{f g} \\
\left(s^{-1}\right)\end{array}$ & $E(R y)$ \\
\hline & & & & & \\
$3 d^{2} 4 p\left({ }^{4} G^{o}\right)$ & $2.09(+8)$ & 0.2728 & $3 d^{2} 4 p\left({ }^{4} D^{o}\right)$ & $2.98(+6)$ & 0.3701 \\
$3 d^{2} 4 p\left({ }^{4} F^{o}\right)$ & $2.30(+8)$ & 0.2835 & $3 d 4 s 4 p\left({ }^{4} D^{o}\right)$ & $2.97(+8)$ & 0.4798 \\
$3 d^{2} 4 p\left({ }^{4} D^{o}\right)$ & $2.54(+8)$ & 0.2979 & $3 d 4 s 4 p\left({ }^{4} F^{o}\right)$ & $8.23(+8)$ & 0.4806 \\
\hline
\end{tabular}

Table 2: States and state-specific recombination rate coefficients $\alpha_{R C}\left(\mathrm{~cm}^{3} \mathrm{sec}^{-1}\right.$ of Ti I, in order of their contributions to the total recombination rates at temperatures 300,2500 , $10^{4}$, and $10^{5} \mathrm{~K}$. Contrary to the usual assumption, the table shows that the ground state, $3 d^{2} 4 s^{2}\left({ }^{3} F\right)$, is not the most dominant contributor at these temperatures.

\begin{tabular}{|c|c|c|c|c|c|c|c|}
\hline \multirow{2}{*}{\multicolumn{2}{|c|}{$\begin{array}{l}\text { State } \\
\mathrm{T}=300 \mathrm{~K}\end{array}$}} & \multirow{2}{*}{\multicolumn{2}{|c|}{$\begin{array}{ll}\text { State } & \\
& 2500\end{array}$}} & State & $\alpha_{R C}$ & State & $\alpha_{R C}$ \\
\hline & & & & \multicolumn{2}{|c|}{$\mathrm{T}=10^{4} \mathrm{~K}$} & \multicolumn{2}{|l|}{$10^{5}$} \\
\hline $3 d 24 s 2 F e 4 p 3 F o$ & $5.94 \mathrm{E}-13$ & $3 d 24 s 2 F e 4 p 3 F o$ & $1.03 \mathrm{E}-13$ & $3 d 34 F e 4 p 3 G o$ & $4.93 \mathrm{E}-14$ & $3 d 34 F e 4 s 5 F e$ & $3.96 \mathrm{E}-14$ \\
\hline $3 d 24 s 4 F e 4 p 3 F o$ & $3.06 \mathrm{E}-13$ & $3 d 24 s 4 F e 4 p 3 F o$ & $9.33 \mathrm{E}-14$ & $3 d 24 s 2 F e 4 p 3 F o$ & $3.48 \mathrm{E}-14$ & $3 d 24 s 4 F e 9 g 5 I e$ & $3.11 \mathrm{E}-14$ \\
\hline $3 d 24 s 4 F e 4 p 3 G o$ & $1.85 \mathrm{E}-13$ & $3 d 34 F e 4 s 5 F e$ & $8.00 \mathrm{E}-14$ & $3 d 34 \mathrm{Fe} 4 s 5 \mathrm{Fe}$ & $3.35 \mathrm{E}-14$ & $3 d 24 s 4 F e 6 g 5 I e$ & $2.55 \mathrm{E}-14$ \\
\hline $3 d 34 \mathrm{Fe} 4 p 3 \mathrm{Do}$ & $1.82 \mathrm{E}-13$ & $3 d 34 F e 4 p 3 G o$ & $6.69 \mathrm{E}-14$ & $3 d 24 s 4 F e 4 p 5 G o$ & $2.93 \mathrm{E}-14$ & $3 d 24 s 4 F e 5 f 5 I o$ & $2.41 \mathrm{E}-14$ \\
\hline $3 d 24 s 23 F e$ & $1.08 \mathrm{E}-13$ & $3 d 32 \mathrm{He} 4 s 3 \mathrm{He}$ & $6.11 \mathrm{E}-14$ & $3 d 24 s 4 F e 4 p 5 F_{o}$ & $2.83 \mathrm{E}-14$ & $3 d 24 p 4 F o 4 f 5 G e$ & $2.13 \mathrm{E}-14$ \\
\hline $3 d 34 F e 4 p 3 F o$ & $9.32 \mathrm{E}-14$ & $3 d 32 \mathrm{He} 4 \mathrm{~s} 3 \mathrm{He}$ & $6.11 \mathrm{E}-14$ & $3 d 24 s 4 F e 5 s 5 F e$ & $2.67 \mathrm{E}-14$ & $3 d 24 s 4 \mathrm{Fe} 9 \mathrm{~g} 5 \mathrm{He}$ & $1.95 \mathrm{E}-14$ \\
\hline $3 d 24 s 2 F e 4 p 3 G o$ & $8.17 \mathrm{E}-14$ & $3 d 34 F e 4 p 3 D_{o}$ & $5.24 \mathrm{E}-14$ & $3 d 24 s 2 D e 4 p 3 F_{o}$ & $2.61 \mathrm{E}-14$ & $3 d 24 s 4 F e 7 g 5 I e$ & $1.85 \mathrm{E}-14$ \\
\hline $3 d 24 s 2 D e 4 p 3 F_{o}$ & $6.54 \mathrm{E}-14$ & $3 d 32 G e 4 s 3 G e$ & $5.04 \mathrm{E}-14$ & $3 d 24 p 4 F o 4 f 5 G e$ & $2.58 \mathrm{E}-14$ & $3 \mathrm{~d} 32 \mathrm{He} 4 \mathrm{~s} 3 \mathrm{He}$ & $1.73 \mathrm{E}-14$ \\
\hline $3 d 24 s 4 F e 4 p 3 D o$ & $6.38 \mathrm{E}-14$ & $3 d 24 s 23 F e$ & $4.40 \mathrm{E}-14$ & $3 d 24 s 4 F e 6 g 5 I e$ & $2.48 \mathrm{E}-14$ & $3 d 32 \mathrm{He} 4 s 3 \mathrm{He}$ & $1.73 \mathrm{E}-14$ \\
\hline $3 d 34 F e 4 p 3 G o$ & $4.89 \mathrm{E}-14$ & $3 d 24 s 4 F e 4 p 3 G o$ & $4.19 \mathrm{E}-14$ & $3 d 32 \mathrm{He} 4 s 3 \mathrm{He}$ & $2.36 \mathrm{E}-14$ & $3 d 24 s 4 F e 7 g 5 H e$ & $1.71 \mathrm{E}-14$ \\
\hline $3 d 24 s 4 F e 4 p 5 G o$ & $4.72 \mathrm{E}-14$ & $3 d 24 s 4 F e 4 p 5 G o$ & $3.80 \mathrm{E}-14$ & $3 d 32 \mathrm{He} 4 s 3 \mathrm{He}$ & $2.36 \mathrm{E}-14$ & $3 d 24 s 4 F e 4 f 5 I o$ & $1.70 \mathrm{E}-14$ \\
\hline $3 d 34 F e 4 p 5 G o$ & $4.32 \mathrm{E}-14$ & $3 d 24 s 4 F e 4 p 5 F o$ & $3.52 \mathrm{E}-14$ & $3 d 24 s 4 F e 5 p 5 G o$ & $2.20 \mathrm{E}-14$ & $3 d 24 s 4 F e 4 p 5 G o$ & $1.67 \mathrm{E}-14$ \\
\hline $3 d 24 s 4 F e 4 p 5 F o$ & $3.85 \mathrm{E}-14$ & $3 d 34 F e 4 p 3 F o$ & $3.47 \mathrm{E}-14$ & $3 d 24 s 4 F e 4 f 5 I o$ & $2.03 \mathrm{E}-14$ & $3 d 24 s 4 F e 7 h 5 K o$ & $1.62 \mathrm{E}-14$ \\
\hline $3 d 34 F e 4 p 5 F o$ & $3.62 \mathrm{E}-14$ & $3 d 24 s 4 F e 4 d 5 F e$ & $3.18 \mathrm{E}-14$ & $3 d 24 s 4 F e 4 p 3 F o$ & $1.97 \mathrm{E}-14$ & $3 d 24 s 4 F e 5 f 5 H o$ & $1.60 \mathrm{E}-14$ \\
\hline $3 d 32 \mathrm{He} 5 \mathrm{~s} 3 \mathrm{He}$ & $3.59 \mathrm{E}-14$ & $3 d 24 s 23 P e$ & $2.88 \mathrm{E}-14$ & $3 d 32 G e 4 s 3 G e$ & $1.94 \mathrm{E}-14$ & $3 d 24 s 4 F e 5 f 5 G o$ & $1.56 \mathrm{E}-14$ \\
\hline $3 d 32 \mathrm{He} 4 \mathrm{~s} 3 \mathrm{He}$ & $3.46 \mathrm{E}-14$ & $3 d 24 s 4 F e 4 p 3 D o$ & $2.82 \mathrm{E}-14$ & $3 d 24 s 4 F e 4 f 5 G o$ & $1.90 \mathrm{E}-14$ & $3 d 24 s 4 F e 7 h 3 K o$ & $1.53 \mathrm{E}-14$ \\
\hline $3 d 32 \mathrm{He} 4 \mathrm{~s} 3 \mathrm{He}$ & $3.46 \mathrm{E}-14$ & $3 d 24 s 2 F e 4 p 3 G o$ & $2.81 \mathrm{E}-14$ & $3 d 24 s 2 F e 4 p 3 G o$ & $1.79 \mathrm{E}-14$ & $3 d 24 s 4 F e 8 f 5 H o$ & $1.49 \mathrm{E}-14$ \\
\hline $3 d 24 s 4 F e 4 p 5 D o$ & $3.43 \mathrm{E}-14$ & $3 d 24 s 2 D e 4 p 3 F o$ & $2.76 \mathrm{E}-14$ & $3 d 34 F e 4 p 3 F o$ & $1.78 \mathrm{E}-14$ & $3 d 32 G e 4 s 3 G e$ & $1.48 \mathrm{E}-14$ \\
\hline $3 d 34 F e 4 s 3 F e$ & $2.81 \mathrm{E}-14$ & $3 d 32 D e 4 s 3 D e$ & $2.46 \mathrm{E}-14$ & $3 d 24 s 23 F e$ & $1.77 \mathrm{E}-14$ & $3 d 24 s 4 F e 7 g 5 G e$ & $1.34 \mathrm{E}-14$ \\
\hline $3 d 24 s 4 P e 4 p 5 D o$ & $2.55 \mathrm{E}-14$ & $3 d 24 s 2 D e 4 s 3 D e$ & $2.30 \mathrm{E}-14$ & $3 d 24 s 4 F e 9 g 5 I e$ & $1.71 \mathrm{E}-14$ & $3 d 24 s 4 F e 4 f 5 G o$ & $1.33 \mathrm{E}-14$ \\
\hline
\end{tabular}

[17] S.N. Nahar, Phys. Rev. A 53, 2417 (1996)

[18] S.N. Nahar, W. Eissner, G.X. Chen, A.K. Pradhan, A\&A 408, 789 (2003)

[19] Y. Yu, M.J. Seaton, J. Phys. B 20, 6409 (1987) 


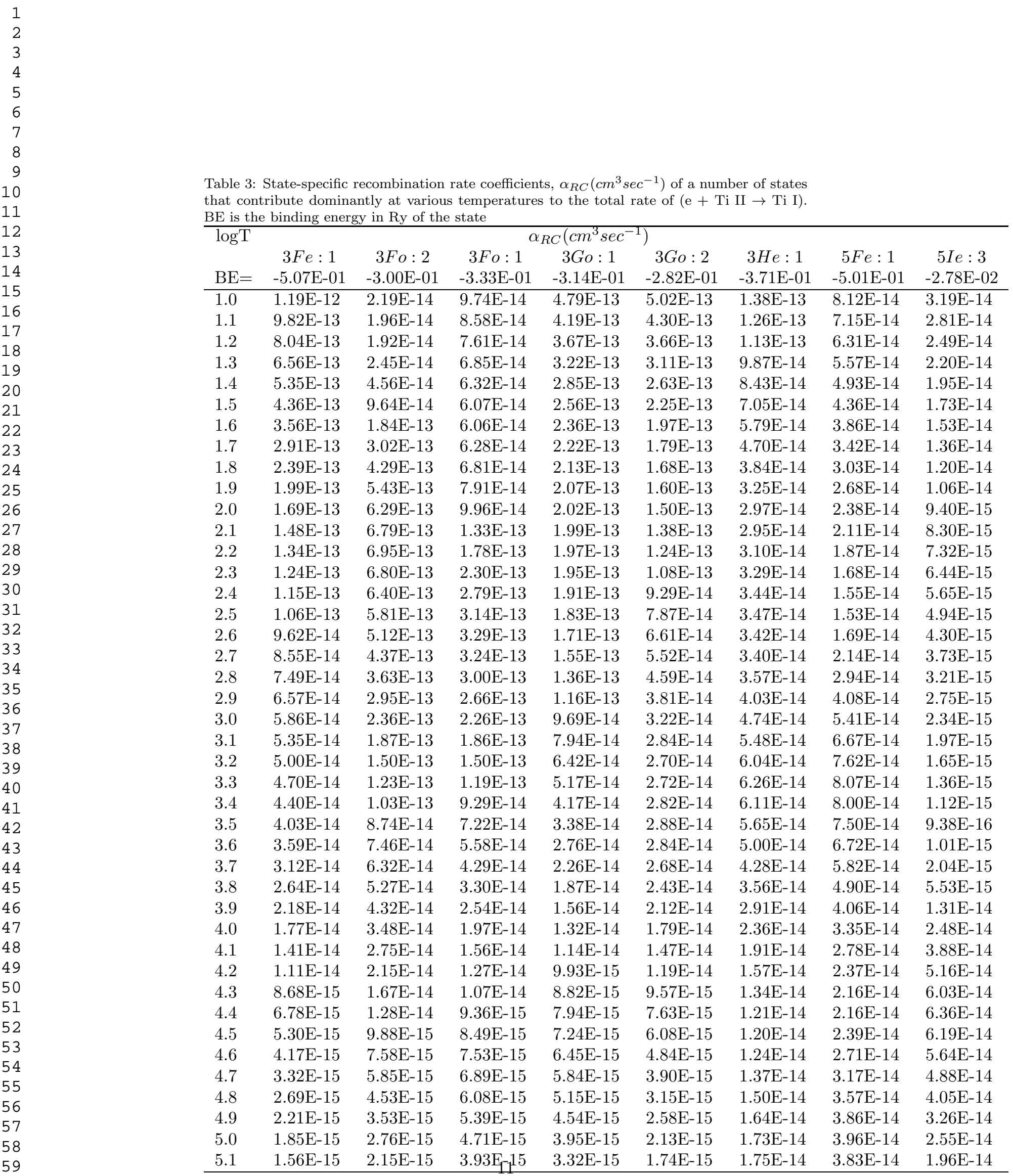




\begin{tabular}{|c|c|c|c|c|c|c|c|c|}
\hline \multirow[t]{2}{*}{$\log \mathrm{T}$} & \multicolumn{8}{|c|}{$\alpha_{R C}\left(\mathrm{~cm}^{3} \sec ^{-1}\right)$} \\
\hline & $3 F e: 1$ & $3 F o: 2$ & $3 F_{O}: 1$ & $3 G o: 1$ & $3 G o: 2$ & $3 H e: 1$ & $5 F e: 1$ & $5 I e: 3$ \\
\hline $\mathrm{BE}=$ & $-5.07 \mathrm{E}-01$ & $-3.00 \mathrm{E}-01$ & $-3.33 \mathrm{E}-01$ & $-3.14 \mathrm{E}-01$ & $-2.82 \mathrm{E}-01$ & $-3.71 \mathrm{E}-01$ & $-5.01 \mathrm{E}-01$ & $-2.78 \mathrm{E}-02$ \\
\hline 5.2 & $1.33 \mathrm{E}-15$ & $1.67 \mathrm{E}-15$ & $3.24 \mathrm{E}-15$ & $2.76 \mathrm{E}-15$ & $1.43 \mathrm{E}-15$ & $1.70 \mathrm{E}-14$ & $3.53 \mathrm{E}-14$ & $1.48 \mathrm{E}-14$ \\
\hline 5.3 & $1.12 \mathrm{E}-15$ & $1.30 \mathrm{E}-15$ & $2.67 \mathrm{E}-15$ & $2.30 \mathrm{E}-15$ & $1.17 \mathrm{E}-15$ & & $3.13 \mathrm{E}-14$ & $1.10 \mathrm{E}-14$ \\
\hline 5.4 & $9.44 \mathrm{E}-16$ & $1.01 \mathrm{E}-15$ & $2.14 \mathrm{E}-15$ & $1.87 \mathrm{E}-15$ & $9.47 \mathrm{E}-16$ & & $2.67 \mathrm{E}-14$ & $8.10 \mathrm{E}-15$ \\
\hline 5.5 & $7.83 \mathrm{E}-16$ & $7.83 \mathrm{E}-16$ & $1.73 \mathrm{E}-15$ & $1.52 \mathrm{E}-15$ & $7.65 \mathrm{E}-16$ & & $2.22 \mathrm{E}-14$ & $5.92 \mathrm{E}-15$ \\
\hline 5.6 & $6.39 \mathrm{E}-16$ & $5.96 \mathrm{E}-16$ & $1.32 \mathrm{E}-15$ & $1.19 \mathrm{E}-15$ & & & & \\
\hline 5.7 & $5.14 \mathrm{E}-16$ & $4.59 \mathrm{E}-16$ & $1.04 \mathrm{E}-15$ & $9.51 \mathrm{E}-16$ & $4.80 \mathrm{E}$ & & & \\
\hline 5.8 & $4.08 \mathrm{E}-16$ & $3.52 \mathrm{E}-16$ & $8.22 \mathrm{E}-16$ & 7.57E-16 & $3.79 \mathrm{E}-16$ & & & $2.24 \mathrm{E}-15$ \\
\hline 5.9 & $3.16 \mathrm{E}-16$ & $2.59 \mathrm{E}-16$ & & $5.50 \mathrm{E}-16$ & 2.87 & & & \\
\hline 6.0 & $2.45 \mathrm{E}-16$ & $1.96 \mathrm{E}-16$ & 4.32 & $4.29 \mathrm{E}-16$ & 2.23 & & & \\
\hline 6.1 & $1.88 \mathrm{E}-16$ & 1.49 & 3.32 & $3.34 \mathrm{E}-16$ & 1.72 & & & \\
\hline 6.2 & $1.42 \mathrm{E}-16$ & $1.11 \mathrm{E}-16$ & 2.47 & 2.54 & 1.3 & & & \\
\hline 6.3 & $1.07 \mathrm{E}-16$ & 8.381 & 1.88 & 1.95 & 1.01 & & & \\
\hline 6.4 & $8.02 \mathrm{E}-17$ & 629 & & 1.50 & & & & \\
\hline 6.5 & $5.97 \mathrm{E}-17$ & 4.71 & 1.0 & 1.14 & 5.8 & & & \\
\hline 6.6 & $4.43 \mathrm{E}-17$ & 353 & & & & & & \\
\hline 6.7 & $3.27 \mathrm{E}-17$ & 7 & & & & & & \\
\hline 6.8 & $2.41 \mathrm{E}-17$ & & & & & & & \\
\hline 6.9 & 1.77 & 7 & & 3.7 & & & & \\
\hline 7.0 & 1.30 & 1.0 & 2.6 & 2.8 & & & & -17 \\
\hline 7.1 & $9.50 \mathrm{H}$ & & & 2.1 & & & & -17 \\
\hline 7.2 & 6.95 & 6.0 & & 1.6 & 7.7 & & & -17 \\
\hline 7.3 & 5.081 & 4.47 & 1.1 & 1.21 & 5.7 & & & -17 \\
\hline 7.4 & $3.71 \mathrm{E}-18$ & & & & 4.3 & & & E-18 \\
\hline 7.5 & $2.71 \mathrm{E}-18$ & 2.47 & 6.7 & 6.92 & 3.2 & & & OE-18 \\
\hline 7.6 & $1.98 \mathrm{E}-18$ & $1.84 \mathrm{E}-18$ & & 5.25 & & & & $2 \mathrm{E}-18$ \\
\hline 7.7 & $1.44 \mathrm{E}-18$ & $1.38 \mathrm{E}-18$ & $4.05 \mathrm{E}-18$ & 3.99E-18 & 1.78 & & & $3.41 \mathrm{E}-18$ \\
\hline 7.8 & $1.05 \mathrm{E}-18$ & $1.03 \mathrm{E}-18$ & & $3.04 \mathrm{E}-18$ & & & & $2.42 \mathrm{E}-18$ \\
\hline 7.9 & $7.72 \mathrm{E}-19$ & $7.75 \mathrm{E}-19$ & $2.49 \mathrm{E}-18$ & $2.33 \mathrm{E}-18$ & $9.97 \mathrm{E}-19$ & $3.00 \mathrm{E}-17$ & $2.15 \mathrm{E}-17$ & $1.72 \mathrm{E}-18$ \\
\hline 8.0 & $5.65 \mathrm{E}-19$ & & & $1.80 \mathrm{E}-18$ & $7.48 \mathrm{E}-19$ & & $1.60 \mathrm{E}-17$ & $1.22 \mathrm{E}-18$ \\
\hline 8.1 & $4.14 \mathrm{E}-19$ & $4.44 \mathrm{E}-19$ & $1.58 \mathrm{E}-18$ & $1.40 \mathrm{E}-18$ & $5.63 \mathrm{E}-19$ & $1.65 \mathrm{E}-17$ & $1.20 \mathrm{E}-17$ & $8.62 \mathrm{E}-19$ \\
\hline 8.2 & $3.04 \mathrm{E}-19$ & & $1.28 \mathrm{E}-18$ & $1.09 \mathrm{E}-18$ & $4.26 \mathrm{E}-19$ & $1.22 \mathrm{E}-17$ & 8.99E-18 & $6.11 \mathrm{E}-19$ \\
\hline 8.3 & $2.24 \mathrm{E}-19$ & $2.60 \mathrm{E}-19$ & $1.04 \mathrm{E}-18$ & 8.62E-19 & $3.24 \mathrm{E}-19$ & $9.09 \mathrm{E}-18$ & $6.80 \mathrm{E}-18$ & $4.33 \mathrm{E}-19$ \\
\hline 8.4 & $1.65 \mathrm{E}-19$ & $2.02 \mathrm{E}-19$ & $8.60 \mathrm{E}-19$ & $6.85 \mathrm{E}-19$ & $2.47 \mathrm{E}-19$ & $6.76 \mathrm{E}-18$ & $5.18 \mathrm{E}-18$ & $3.07 \mathrm{E}-19$ \\
\hline 8.5 & $1.23 \mathrm{E}-19$ & $1.58 \mathrm{E}-19$ & $7.14 \mathrm{E}-19$ & $5.49 \mathrm{E}-19$ & $1.90 \mathrm{E}-19$ & $5.03 \mathrm{E}-18$ & $3.97 \mathrm{E}-18$ & $2.17 \mathrm{E}-19$ \\
\hline 8.6 & $9.09 \mathrm{E}-20$ & $1.24 \mathrm{E}-19$ & $5.99 \mathrm{E}-19$ & $4.45 \mathrm{E}-19$ & $1.48 \mathrm{E}-19$ & $3.76 \mathrm{E}-18$ & $3.07 \mathrm{E}-18$ & $1.54 \mathrm{E}-19$ \\
\hline 8.7 & $6.81 \mathrm{E}-20$ & $9.86 \mathrm{E}-20$ & $5.06 \mathrm{E}-19$ & $3.66 \mathrm{E}-19$ & $1.15 \mathrm{E}-19$ & $2.81 \mathrm{E}-18$ & $2.40 \mathrm{E}-18$ & $1.09 \mathrm{E}-19$ \\
\hline 8.8 & $5.19 \mathrm{E}-20$ & $7.92 \mathrm{E}-20$ & $4.31 \mathrm{E}-19$ & $3.03 \mathrm{E}-19$ & $9.07 \mathrm{E}-20$ & $2.12 \mathrm{E}-18$ & $1.93 \mathrm{E}-18$ & $7.74 \mathrm{E}-20$ \\
\hline 8.9 & $3.96 \mathrm{E}-20$ & $6.43 \mathrm{E}-20$ & $3.71 \mathrm{E}-19$ & $2.54 \mathrm{E}-19$ & $7.21 \mathrm{E}-20$ & $1.60 \mathrm{E}-18$ & $1.52 \mathrm{E}-18$ & $5.49 \mathrm{E}-20$ \\
\hline 9.0 & $3.04 \mathrm{E}-20$ & $5.30 \mathrm{E}-20$ & $3.20 \mathrm{E}-19$ & 2.12E-19 & $5.85 \mathrm{E}-20$ & $1.22 \mathrm{E}-18$ & $1.23 \mathrm{E}-18$ & $3.89 \mathrm{E}-20$ \\
\hline
\end{tabular}


Table 4: Total recombination rate coefficients, $\alpha_{R C}\left(\mathrm{~cm}^{3} \mathrm{sec}^{-1}\right)$ wuth temperature for $(\mathrm{e}+$ Ti II $\rightarrow$ Ti I).

\begin{tabular}{|c|c|c|c|c|c|}
\hline $\begin{array}{c}\log \mathrm{T} \\
(\mathrm{K})\end{array}$ & $\begin{array}{c}\alpha_{R} \\
\left(\mathrm{~cm}^{3} \sec ^{-1}\right)\end{array}$ & $\begin{array}{c}\log \mathrm{T} \\
(\mathrm{K})\end{array}$ & $\begin{array}{c}\alpha_{R} \\
\left(\mathrm{~cm}^{3} \sec ^{-1}\right)\end{array}$ & $\begin{array}{c}\log \mathrm{T} \\
(\mathrm{K})\end{array}$ & $\begin{array}{c}\alpha_{R} \\
\left(\mathrm{~cm}^{3} \sec ^{-1}\right)\end{array}$ \\
\hline 1.0 & $2.89 \mathrm{E}-11$ & 3.7 & $1.52 \mathrm{E}-12$ & 6.4 & $1.94 \mathrm{E}-13$ \\
\hline 1.1 & $2.47 \mathrm{E}-11$ & 3.8 & $2.15 \mathrm{E}-12$ & 6.5 & $1.39 \mathrm{E}-13$ \\
\hline 1.2 & $2.11 \mathrm{E}-11$ & 3.9 & $3.95 \mathrm{E}-12$ & 6.6 & $9.94 \mathrm{E}-14$ \\
\hline 1.3 & $1.81 \mathrm{E}-11$ & 4.0 & $7.39 \mathrm{E}-12$ & 6.7 & $7.10 \mathrm{E}-14$ \\
\hline 1.4 & $1.55 \mathrm{E}-11$ & 4.1 & $1.23 \mathrm{E}-11$ & 6.8 & $5.07 \mathrm{E}-14$ \\
\hline 1.5 & $1.33 \mathrm{E}-11$ & 4.2 & $1.77 \mathrm{E}-11$ & 6.9 & $3.62 \mathrm{E}-14$ \\
\hline 1.6 & $1.15 \mathrm{E}-11$ & 4.3 & $2.24 \mathrm{E}-11$ & 7.0 & $2.59 \mathrm{E}-14$ \\
\hline 1.7 & $1.01 \mathrm{E}-11$ & 4.4 & $2.56 \mathrm{E}-11$ & 7.1 & $1.85 \mathrm{E}-14$ \\
\hline 1.8 & $8.91 \mathrm{E}-12$ & 4.5 & $2.66 \mathrm{E}-11$ & 7.2 & $1.32 \mathrm{E}-14$ \\
\hline 1.9 & $7.95 \mathrm{E}-12$ & 4.6 & $2.58 \mathrm{E}-11$ & 7.3 & $9.41 \mathrm{E}-15$ \\
\hline 2.0 & 7.13E-12 & 4.7 & $2.35 \mathrm{E}-11$ & 7.4 & $6.72 \mathrm{E}-15$ \\
\hline 2.1 & $6.44 \mathrm{E}-12$ & 4.8 & $2.05 \mathrm{E}-11$ & 7.5 & $4.80 \mathrm{E}-15$ \\
\hline 2.2 & $5.84 \mathrm{E}-12$ & 4.9 & $1.71 \mathrm{E}-11$ & 7.6 & $3.43 \mathrm{E}-15$ \\
\hline 2.3 & $5.33 \mathrm{E}-12$ & 5.0 & $1.38 \mathrm{E}-11$ & 7.7 & $2.45 \mathrm{E}-15$ \\
\hline 2.4 & $4.86 \mathrm{E}-12$ & 5.1 & $1.09 \mathrm{E}-11$ & 7.8 & $1.75 \mathrm{E}-15$ \\
\hline 2.5 & $4.42 \mathrm{E}-12$ & 5.2 & $8.44 \mathrm{E}-12$ & 7.9 & $1.25 \mathrm{E}-15$ \\
\hline 2.6 & $3.99 \mathrm{E}-12$ & 5.3 & $6.42 \mathrm{E}-12$ & 8.0 & $8.98 \mathrm{E}-16$ \\
\hline 2.7 & $3.56 \mathrm{E}-12$ & 5.4 & $4.82 \mathrm{E}-12$ & 8.1 & $6.45 \mathrm{E}-16$ \\
\hline 2.8 & $3.16 \mathrm{E}-12$ & 5.5 & $3.58 \mathrm{E}-12$ & 8.2 & $4.63 \mathrm{E}-16$ \\
\hline 2.9 & $2.78 \mathrm{E}-12$ & 5.6 & $2.63 \mathrm{E}-12$ & 8.3 & $3.33 \mathrm{E}-16$ \\
\hline 3.0 & $2.46 \mathrm{E}-12$ & 5.7 & $1.92 \mathrm{E}-12$ & 8.4 & $2.41 \mathrm{E}-16$ \\
\hline 3.1 & $2.20 \mathrm{E}-12$ & 5.8 & $1.40 \mathrm{E}-12$ & 8.5 & $1.74 \mathrm{E}-16$ \\
\hline 3.2 & $1.99 \mathrm{E}-12$ & 5.9 & $1.01 \mathrm{E}-12$ & 8.6 & $1.27 \mathrm{E}-16$ \\
\hline 3.3 & $1.83 \mathrm{E}-12$ & 6.0 & $7.31 \mathrm{E}-13$ & 8.7 & $9.25 \mathrm{E}-17$ \\
\hline 3.4 & $1.68 \mathrm{E}-12$ & 6.1 & $5.26 \mathrm{E}-13$ & 8.8 & $6.79 \mathrm{E}-17$ \\
\hline 3.5 & $1.55 \mathrm{E}-12$ & 6.2 & $3.78 \mathrm{E}-13$ & 8.9 & $5.01 \mathrm{E}-17$ \\
\hline 3.6 & $1.46 \mathrm{E}-12$ & 6.3 & $2.71 \mathrm{E}-13$ & 9.0 & $3.74 \mathrm{E}-17$ \\
\hline
\end{tabular}


Figure 1: Partial photoionization cross sections, $\sigma_{P I}$, of the (a) ground $3 d^{2} 4 s^{2}\left({ }^{3} F\right)$ and (b) excited $3 d^{2} 4 s^{2} F 4 p\left(y^{3} F^{o}\right)$ states of Ti I illustrating positions of prominent resonances in the low energy region and background structures that will determine the contributions to the state-specific recombination rate coefficients.

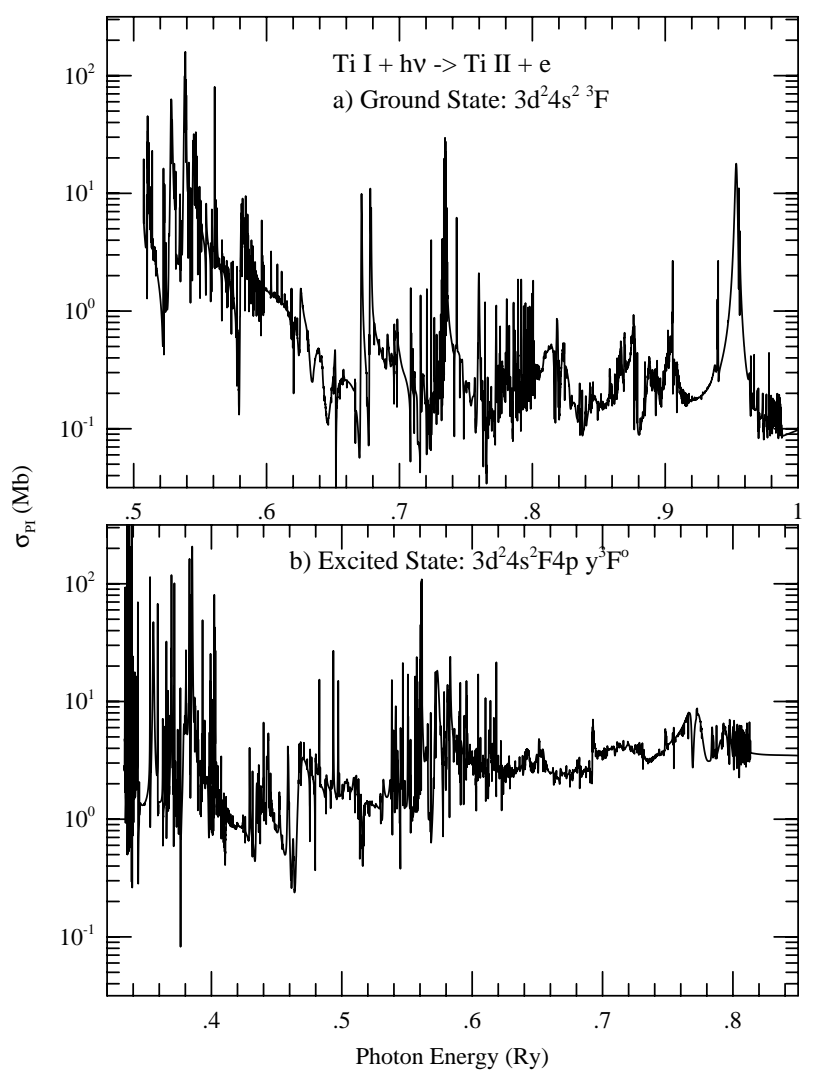


Figure 2: Partial photoionization cross sections, $\sigma_{P I}$, of single outer electron excited states of Ti I: (a) $3 d^{2} 4 s^{4} F 4 p\left(z^{3} G^{o}\right)$, (b) $3 d^{2} 4 s^{4} F 5 s\left(b^{5} F\right)$ demonstrating huge rise in background due to prominent Seaton or PEC (photo-excitation=of-core) resonances (energy positions pointed by arrows). It may be noted that PEC positions do not change energy positions with the ionization energy of the state. 
Figure 3: State-specific recombination rate coefficients $\alpha_{R}(n L S)$, which include contributions of both RR and DR, of ground (a) and a number of excited states (b-g) of Ti I featuring dominance of recombination with temperatures which varies with the states. The variation is due to the positions of strong resonances and enhancements in background cross sections in photoionization. 
Figure 4: Total unified recombination rate coefficients of (e + Ti II $\rightarrow$ Ti I) showing typical features of high recombination at low temperature and DR bump at $\mathrm{T}=25,000 \mathrm{~K}$. It also shows a small hump at a lower temperature around $280 \mathrm{~K}$ due to dominance of low energy resonances in photoionization cross sections. 
Figure 5: Unified total recombination cross sections $\sigma_{R C}$ (top panel a) and rate coefficients $\alpha_{R}$ (lower panel b) of $(\mathrm{e}+\mathrm{Ti}$ II $\rightarrow$ Ti I) with photoelectron energy. Arrows points converging threshold ${ }^{4}(G F D)^{\circ}$ where DR peaks and then drops almost to zero. 